\title{
Preface: Eli Sercarz Memorial Issue, Part II
}

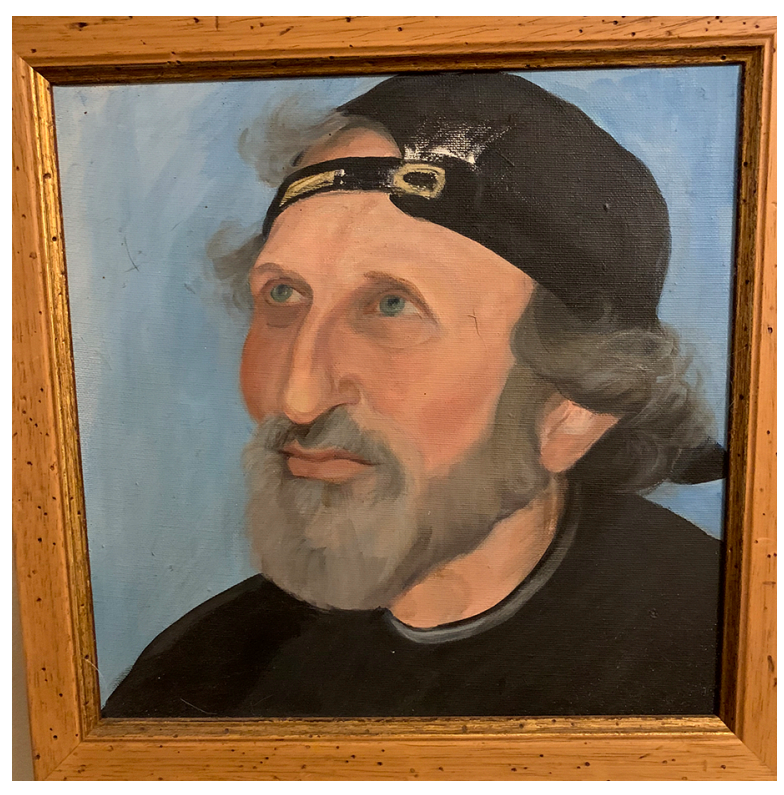

The late Eli E. Sercarz devoted his life and career to the field of immunology, and he systematically dissected several of the unknown fundamental principles and molecular mechanisms that govern the complex immune response mediated by immune cells (T, B, APC). His research investigations and approaches were novel and creative, delineating the means by which specific amino acid sequences of protein molecules dictate a response (immunogenic) or nonresponsiveness (tolerogen) via sophisticated interactions among the APC, T lymphocyte subsets, and B cells. Such meticulous analyses using various protein molecules as models resulted in clarifications of the observed immune defects involved in various human diseases (autoimmune, transplantation, etc.). Eli's guidance and traineeships emanated in hundreds of publications and nurtured the education and development of a large number of academic immunologists, whose careers and research investigations were the direct extensions of his fundamental research.

Eli died at the age of 75 after battling renal cancer. This is the first publication celebrating his memory and legacy. Vipin Kumar and Benjamin Bonavida, guest editors for these special issues in Critical Reviews $^{\mathrm{TM}}$ in Immunology (CRI), were previously Eli's fellows and decided to dedicate three issues in his memory. As anticipated, many of Eli's former fellows and collaborators have responded enthusiastically about dedicating their work in these three issues of CRI, published simultaneously in his memory.

In the second CRI issue, there are nine contributions, outlined briefly as follows:

Celada's memorial article entitled "Homage to Eli Sercarz" describes his time spent in Eli's laboratory during a sabbatical, and he summarizes their relationship by writing, "Best friend, colleague, collaborator, and ultimately co-grandfather of two boys, Nicola and Matteo, Eli Sercarz has enriched my life in many ways."

A second memorial piece by Celada, entitled "Riding with Eli: The Incurable Passion for Searching," describes his various interactions with Eli on public transportation, at restaurants, and in Eli's home, among other places, along with their various discussions on the philosophical aspects of immunology. This memorial is well written and highlights Eli's ingenuity and friendly interactions with Celada and other fellow immunologists.

Peeyush et al.'s article entitled "PRMT5 and Tip60 Modify FOXP3 Function in Tumor Immunity" focuses on posttranslational modifications (PTMs) mediated by protein methyltransferases (PRMTs) (arginine methylation), due to their important roles in several diseases. Among the PRMTs, PRMT5 (type II arginine methyltransferase) is involved in cancer progression. Significantly, the inhibition of RPMT5 results in the modulation of both Tip60 (histone acetyl transferase) activity and FOXP3 levels, thus enhancing antitumor immunity. The review also discusses the molecular interactions between PRMT5 and FOXP3. Inhibitors of PRMT5 are used in clinical trials today.

Kugler-Umana and Swain's article entitled "Understanding the Heterogeneous Population of Age-Associated B Cells and Their Contribution to Autoimmunity and Immune Response to Pathogens" 
reviews the age-associated changes in both innate and adaptive immunities. The authors describe the new population of age-associated $B$ cells $(A B C)$ with a distinct phenotype. They also describe the phenotypes of naïve and activated $\mathrm{ABC}$ cells, along with their functions. In autoimmunity, the antigen-activated $A B C$ cells increase in number. The naïve $A B C$ cells respond to pathogens through the stimulation of TLRs. These findings suggest an important role for naïve and activated ABC cells in infections and autoimmunity, respectively, in the elderly.

Krzych and Althubaiti's article entitled "The Form of an Antigen and Its Molecular Context Do Matter: Infectious Versus Attenuated Plasmodium Sporozoite" reviews the opposite immunological outcomes that occur when subjects are infected with live protozoan parasites versus their attenuated forms: the live form leads to infection, whereas immunization with radiation and genetically attenuated forms induces a durable protective immunity. Infectious sporozoites escape the immune system, whereas exposure to the attenuated forms induces antibodies, CD4 T cells, and memory CD8 T cells. Krzych and Althubaiti discuss the mechanisms by which the sporozoites interact with the antigen-presenting cells, along with the subsequent processing and presentation that should lead to the development of a malarial vaccine in the near future.

Krzych's memorial article "Eli's Propinquity Is Far-Reaching: From E. coli Beta-Galactosidase to the Plasmodium Parasite," describes the author's relationship with Eli as a postdoctoral fellow and her introduction to the antigenicity/immunogenicity of beta-galactosidase. She writes: "The concept of antigen processing and presentation was not yet experimentally verified, but nonetheless, our results suggested some form of antigen degradation by which multiple determinants of an antigen would be presented by different MHC molecules to T cells. These observations have formed my thinking about the immune responses to antigens."

Moudgil's article entitled "Viewing Autoimmune Pathogenesis from the Perspective of Antigen Processing and Determinant Hierarchy" reviews the immunogenicity of Eli's initial discovery of the "cryptic" epitopes on self-antigens that escaped tolerance, which, under inflammatory and immune activation, are presented by the APCs to induce an autoimmune response. This is also followed by "epitope spreading" that leads to the enhancement and propagation of autoimmunity.

Dai's memorial "Drivers and Passengers in T-cell-mediated Autoimmunity-A Tribute to Eli Sercarz" describes his long association with Eli, spanning many years. Briefly, Dai describes the various projects that he undertook, namely, on the myelin basic protein (MBP) and the glutamic acid decarboxylase-65 (GAD65) in type 1 diabetes (TID) by examining driver clones, a concept originated by Eli. Dai recalls: "I had spent about eight years accompanying [Eli] to finish the last part of his journey, including a memorable trip to Crete, Greece, in October 2009. That experience is a special gift that I will hold precious for the rest of my life, not only for those complex beautifully knotted immunology concepts that Eli taught me and the creative projects that we worked on together, but also for the plethora of warm-hearted personal interactions between a mentor and a trainee: the trips, long drives, delicious food, ping-pong games, and his country house in Topanga."

Bonavida's memorial "In Memorium: Vivid Memories of Sercarz's Traineeship and Indebted for my Professional Career" describes his early association with Eli as one of the first PhD students in his laboratory upon Eli's arrival at UCLA. Bonavida's $\mathrm{PhD}$ thesis was on mapping the epitopes of hen egg white lysozyme (HEL), along with the studies that originated many of the descriptive epitopes coined by Eli, such as localized epitopes, strong and weak epitopes, heterotopes, hybrid epitopes, cryptotopes, and heteroclitic epitopes. HEL was a solid model for investigation due to its pure form and known amino acid sequence, along with the sequence of its digested fragments. In addition, another project by Bonavida dealt with tear lysozyme from rabbits, exploring the antibodies derived from systemic immunization and the characterization of human tear proteins, with the identification of a new protein, specific tear prealbumin. Bonavida also describes his close relationship and experience with Eli and 
his gratitude for Eli's influence on his professional career.

The provided reviews discuss up-to-date studies on various immune system topics, several of which resulted from expansions of original findings from Eli's laboratory. The contents of this CRI issue also emphasize the many outcomes of Eli's significant conceptual contributions for managing human diseases today.
Guest Editors:

Vipin Kumar

University of California San Diego

La Jolla, CA, USA

Benjamin Bonavida

University of California, Los Angeles

Los Angeles, CA, USA 